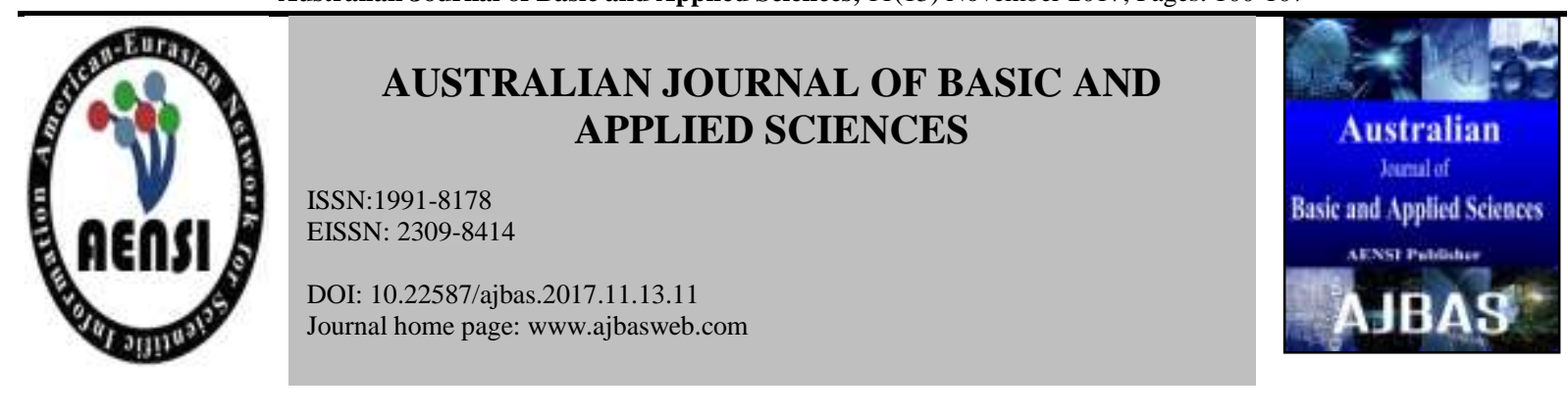

\title{
Dynamic Routing Nodes For Underwater Wireless Sensor Network
}

\author{
${ }^{1}$ J. Paramesh and ${ }^{2}$ C. R. Rene Robin
}

${ }^{1}$ Associate Professor, Department of Information Technology, Misrimal Navajee Munoth Jain Engineering College, Chennai -97, Tamil Nadu, India.

${ }^{2}$ Professor, Department of Computer Science and Engineering, Jerusalem College of Engineering, Chennai-100, Tamil Nadu, India.

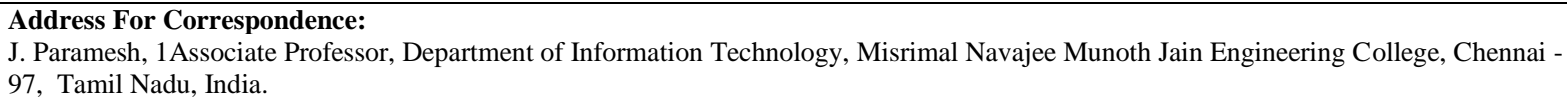

\section{ARTICLE INFO}

\section{Article history:}

Received 19 August 2017

Accepted 1 November 2017

Available online 11 November 2017

Keywords:

Wireless sensor network, underwater WSN,

\begin{abstract}
A B S T R A C T
Underwater wireless communications can enable many scientific, environmental, commercial, safety, and military applications. Wireless signal transmission is also critical to remotely control instruments in ocean observatories and to allow coordination of swarms of autonomous underwater vehicles. In the terrestrial environment, radio waves are used for data transmission whereas in an underwater environment acoustic waves are used for propagation due to their low attenuation. In our proposed system, transmission of data is achieved in an energy efficient manner by the principle of dynamic routing. Since the nodes at the higher depth are placed far away from the surface sink, they find it difficult to send their data efficiently to the surface sink. The dynamic routing concept compares the threshold value of all the nodes and then chooses the particular node at runtime irrespective of depth and forwards the data to the sink. This, in turn, decreases the energy consumption of node.
\end{abstract}

\section{INTRODUCTION}

Wireless communication is the transfer of information between two or more points that are not connected by an electrical conductor. The most common wireless technologies use the radio. With radio waves, distances can be short, such as a few meters for television or as far as thousands or even millions of kilometer for deepspace radio communications (Pierre Viland et al., 2009). It encompasses various types of fixed, mobile, and portable applications, including two-way radios, cellular telephones, personal digital assistants (PDAs), and wireless networking (Thi Minh Hien Ngo, Gheorghe Zaharia, 2007; Murat Uysal and Costas N. Georghiades, 2000). Other examples of applications of radio wireless technology include GPS units, garage door openers, wireless computer mice, keyboards and headsets, headphones, radio receivers, satellite television, broadcast television and cordless telephones.

WSN covers a wide spectrum of military and hospital monitoring, military surveillance, fire and intruder detection and animal tracking. In recent years, new technologies have shaped numerous military and commercial strategies in an unprecedented way. The Wireless Sensor Network (WSN) technology is one such technology and has been attracting significant attention. WSN provide a promising infrastructure for gathering information about parameters of the physical world which can be subsequently processed for both commercial and military applications (Magadevi, N. et al;., 2016; Pritto Paul, P., et al., 2016; Kanagalakshmi, A., et al., 2016; Senthil Murugan, M. and T. Sasilatha, 2016; Divya, S. and K. Stella, 2016). About more than $70 \%$ of the Earth's surface is surrounded by aquatic environment which corresponds to mostly unexplored area. Underwater wireless sensor networks have recently been proposed as an innovative way to observe and explore these harsh environments. However, the efficient data delivery is still a challenging issue in these networks because of the impairments of the acoustic transmission (Anandalatchoumy, S. and G. Sivaradje, 2016; Narender, M. and K.R.

\footnotetext{
Open Access Journal

Published BY AENSI Publication
}

(C) 2017 AENSI Publisher All rights reserved

This work is licensed under the Creative Commons Attribution International License (CC BY). http://creativecommons.org/licenses/by/4.0/

\section{Open Access}

To Cite This Article: J. Paramesh and C. R. Rene Robin., Dynamic Routing Nodes For Underwater Wireless Sensor Network. Aust. J. Basic \& Appl. Sci., 11(13): 100-107, 2017 
Anjana Devi, 2016; Lissy, D. and S. Behin Sam, 2016; Arun Chakravarthy, R. and DR. S. Palaniswami, 2016; Balamurugan, M. and Dr.P. Manimegalai, 2016).

\section{Underwater Wireless Communication:}

Underwater wireless communication is the wireless communication in which acoustic signals (waves) carry digital information through an underwater channel. Electromagnetic waves are not used as they propagate over short distances. Over the past decades, heavy cables were used to establish a high-speed communication between remote end and the surface (Ayaz et al., 2011; Ravindranath Vadlamudi, Dr Syed Umar, 2013; Thumpi, R., et al., 2013; Muhammad Ayaz, et al., 2011; Upena Dalal, 2009). To overcome such difficulties, underwater wireless communication has come into existence.

\subsection{Applications of Underwater Communications:}

- Seismic monitoring: It is used for the scientific study of earthquakes and tsunami.

- Pollution monitoring: They are used to monitor the pollution that occurs due to the noise in the acoustic environment.

- Ocean currents are monitoring: An ocean current is a continuous, directed movement of seawater generated by forces.

- $\quad$ Equipment monitoring and control: It is used to monitor the communication channels used.

- Autonomous Underwater Vehicles (AUV): It is a robot which travels underwater without requiring input from an operator.

- Remotely operated vehicle: A remotely operated underwater vehicle, commonly referred to as an ROV, is a tethered underwater vehicle.

\section{Objectives Of The Work:}

The main aim of this project is to reduce the energy consumption of the node while transferring the data and also to make sure that the data reached the surface sink in an underwater acoustic environment. The mobility of data plays a vital role in underwater sensor networks as they have different levels of depth. The routing techniques deployed in the underwater area is based on the principle that the data to be passed to the sensor that is available at higher depth. They make use of acoustic waves for communication. In our proposed system, the mobility of nodes is achieved in an efficient manner and also by reducing the energy consumption. The nodes at the higher depth find it difficult to send their data efficiently to the surface sink. The neighbouring nodes receive data from nodes at higher depth and pass it to the surface sink. The main advantage is that there will be less possibility that the data getting lost and the surface sink also have the storage of the data's received. The network attains the stability position only if every node on the network participates and contributes effectively for the data to reach the surface sink (Hai Yan, et al., 2010; Jafri, M.R., et al., 2013; Javaid, N., et al., 2013; Jian-feng yan, yuan-liu liu, 2011).

To overcome the problem faced by the existing system we propose the new system in which every node has equal contribution to transfer the data to the surface sink. In our proposed system there is no such condition in which the data should be transferred in a sequence manner within the depth region considered. If the node does not have enough energy to transfer the data to surface sink, they forward the data to the neighbouring node available at different depth. The surface sink once it receives the data stores it in the database for reliability (Tahir, M., et al., 2013; Ian, F., et al., 2006; Theodore, S., Rappaport, 2002).

\section{Hardware And Software Requirement: \\ 4.1. Hardware Requirement:}

- Processor: Intel ${ }^{\circledR}$ core $^{\mathrm{TM}}$ i3 CPU, Hard Disk: 400 GB and RAM: 4 GB

\subsection{Software Requirement:}

- $\quad$ Operating system: Windows XP / Windows 7/Windows 8.

- Language: Java 1.7., Front end Tool: Net Beans IDE, Back end Tool: MySQL

\section{Architecture:}




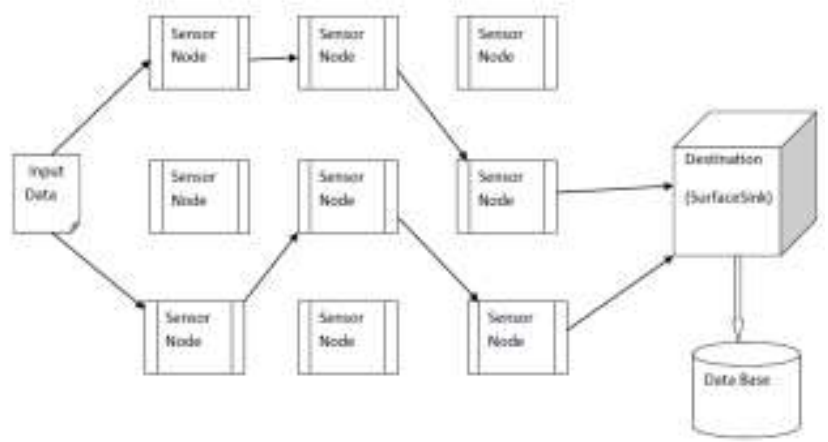

Fig. 1: Proposed System Architecture

\section{Data Flow Diagram:}

The first step is to draw a data flow diagram (DFD). The DFD was first developed by Larry Constantine as a way of expressing system requirements in graphical form. A DFD shows what kind of information will be input to and output from the system, where the data will come from and go to, and where the data will be stored (Murat Uysal, and Costas N. Georghiades, 2004; Pillai, J.N. and S.H. Mneney, 2004; Helmut Bolcskei and Arogyaswami J. Paulraj, 2000; Siavash, M. Alamouti, 1998). It does not show information about the timing of processes, or information about whether processes will operate in sequence or in parallel which is shown in flowchart

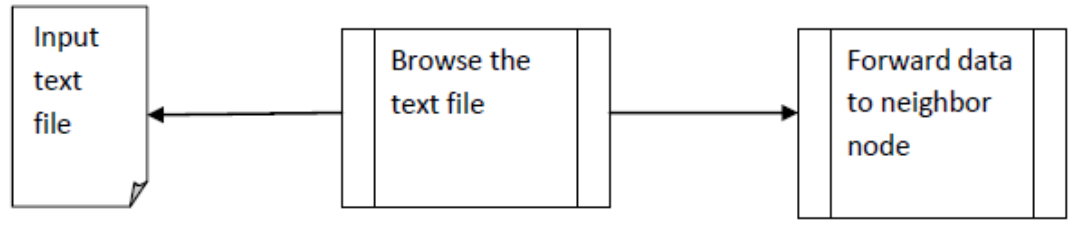

Fig. 2: Level 1: Node Receiving Data for the First Time

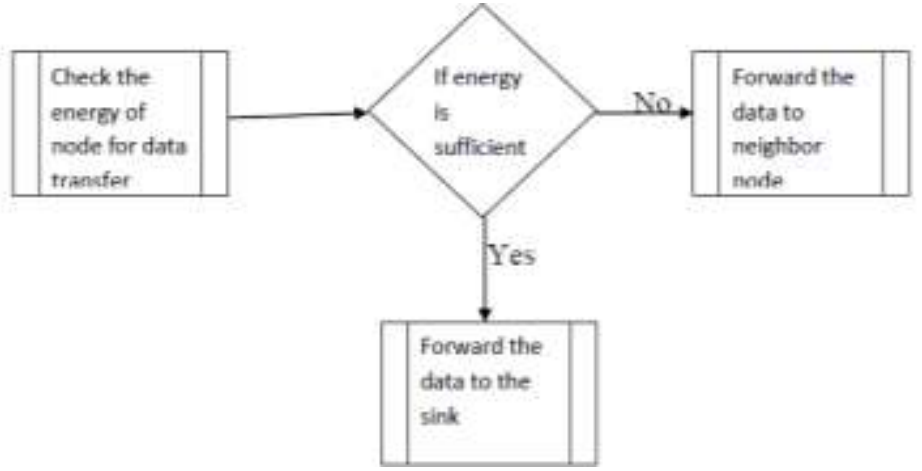

Fig. 3: Level 2: Node Checking its Energy Capability

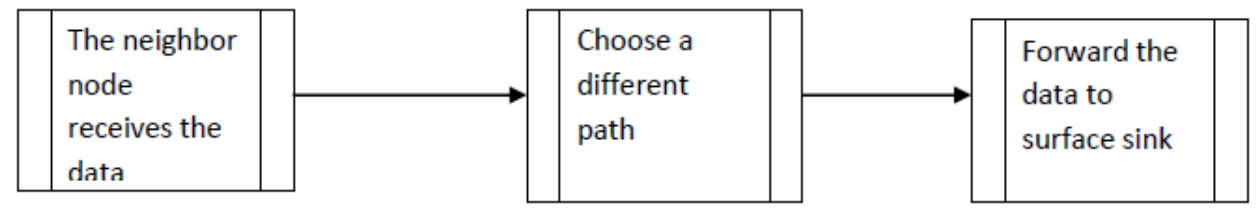

Fig. 4: Level 3: Forwarding Data to the Sink

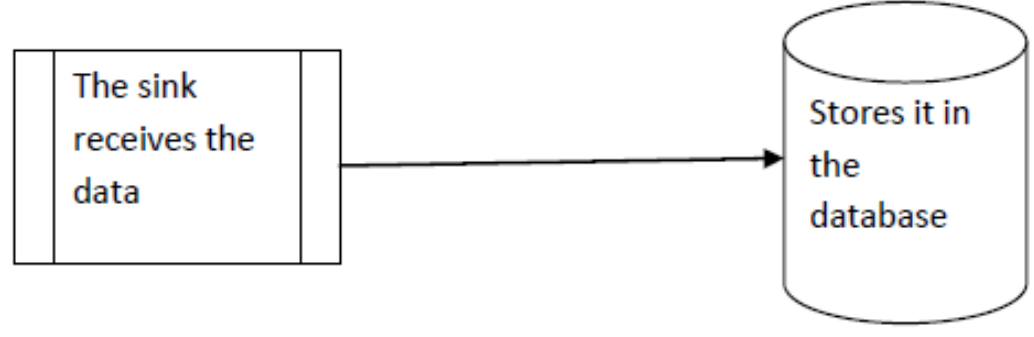

Fig. 5: Level 4: Storing in the Database 


\section{Modules:}

- The proposed system works with the following five modules:

- Network initialization

- Data transmission

- Data routing phase

- Mobility of nodes

- Database storage

\subsection{Network Initialization phase:}

- The first module in our proposed system is network initialization in which the numbers of sensor nodes in the network are decided.

- The nodes are placed in the varying three levels each one representing different depths.

- The nodes at the bottom area are the ones that have higher depth and have minimal noise involved in it. They are placed far from the surface sink.

- The nodes are arranged by the depth value of each.

- Two nodes that have a wide variance in their depth values cannot be the neighbor to each other (Refer Fig. 6).

\subsection{Data Transmission Phase:}

- The second phase of our system is the data transmission phase in which the system starts the actual data transfer.

- The source file that has to be sent to the sink is chosen, and it is sent.

- The source node is the one that receives the input data and checks it energy capability to the forward the data.

- The nodes in the network are desired to transfer the data to the destination

- Nodes in the network have the capability to act as both sender and receiver of the data.

- The node that forwards the data to the destination is the one that is located nearer to the server.

- The data can be forwarded by a node based on the energy allotted to it

- The nodes in the network knows the depth information of other neighboring nodes

- The approach used here is receiver based approach due to the unawareness of the location of the sink.

- The eligible nodes alone forward the data to the surface sink

- The threshold value is considered for a node to act as an eligible one (Refer Fig. 7)

\subsection{Data Routing phase:} destination.

- The third phase of our system is data routing phase in which the data is routed from the source to the

- The concept of routing used here is on-demand data routing.

- The on-demand data routing is the one in which the routing occurs only when it is needed.

- In the existing system, the nodes transfer in the sequence manner within the depth region

- In the proposed work there is no sequence in the transfer of data not only within the depth but also in the network.

- The node first receives the input file and checks its energy whether it can transmit the data.

- If the energy is sufficient enough to transmit the data to the nodes at other depth, then it proceeds.

- If it is not sufficient enough to transfer the data, then it forwards it to the neighboring node.

- The energy of the node may get reduced every time it transfers the data (Refer Fig. 8)

\subsection{Mobility of data:}

- The fourth phase is the one in which the mobility of data takes place.

- The mobility refers to the actual movement of nodes.

- The node, once it receives the data, checks its residual energy for forwarding the data.

- If the energy is not enough, then it is forwarded to the data within the network.

- The node does not restrict itself to transfer the data only within its depth, but if it can reach to any other node of different depth, then it transfers it.

- Any node can play two roles here that are sender and receiver.

- A node once it receives the data makes sure that it does not hold the data for a longer time.

- The holding time of a node must be smaller so that the network stability is maintained.

- If a node transfers the data and found that it does not reach correctly, then it sends the data to the other node by choosing a different path. 
- Once the different path is chosen, then the data may reach the surface sink, and this happens with all the nodes in the network.

- Now the data reaches the surface sink in a reliable manner. (Refer Fig. 9)

\subsection{Database storage:}

- The last phase of our system is that the data gets stored in the surface sink

- The server receives the data and have the backup of the file in its storage

- The tool used here is the MYSQL, and they store the actual data that is transferred

- The database contains the unique id for each data transfer, the actual data sent, and the time at which the data is sent

\section{Testing And Implementation:}

System testing ensures that the entire integrated software system meets requirements. It tests a configuration to ensure known and predictable results. System testing is based on process descriptions and flows, emphasizing pre-driven process links and integration points. Interfacing systems or procedures must be invoked. Organization and preparation of functional tests is focused on requirements, key functions, or special test cases. Also, systematic coverage about identify Business process flows; data fields, predefined processes, and successive processes must be considered for testing. Before functional testing is complete, additional tests are identified and the effective value of current tests is determined.

\section{Experimental Results And Analysis:}

In our proposed system, adding the functionalities of cluster head to a node is to reduce the energy consumption, transmission loss and propagation loss. The energy consumption is achieved in our proposed system by using dynamic routing. Dynamic routing is the concept, in which the transmission path is determined randomly at the runtime by considering the threshold values of nodes. The existing system makes use of ondemand data routing, and the threshold values are determined at the runtime, but the transmission path is predefined. This in turn increases the energy consumption of nodes. Thus we conclude that providing the cluster head functionality and dynamic routing makes the data propagation more efficient in an aqueous environment.

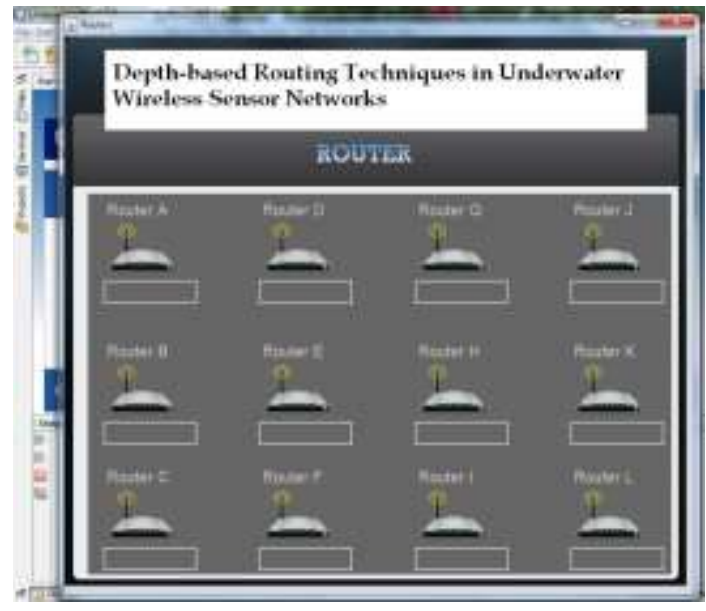

Fig. 6: Network Initialization

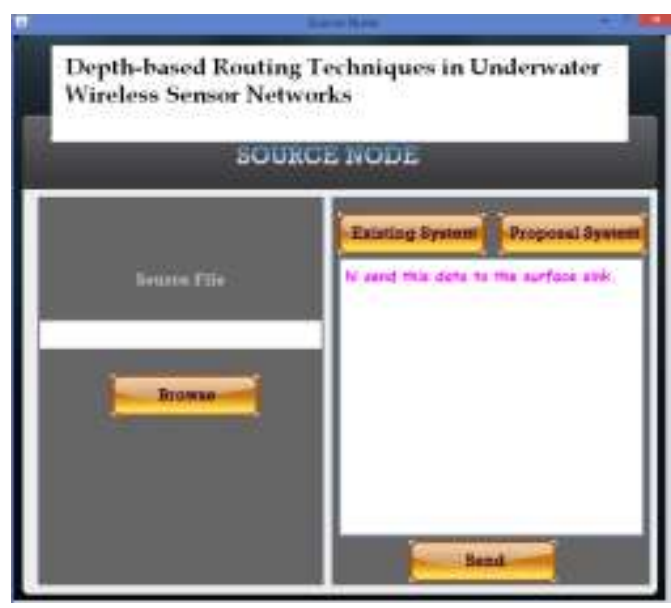

Fig. 7: Sending file to sink 


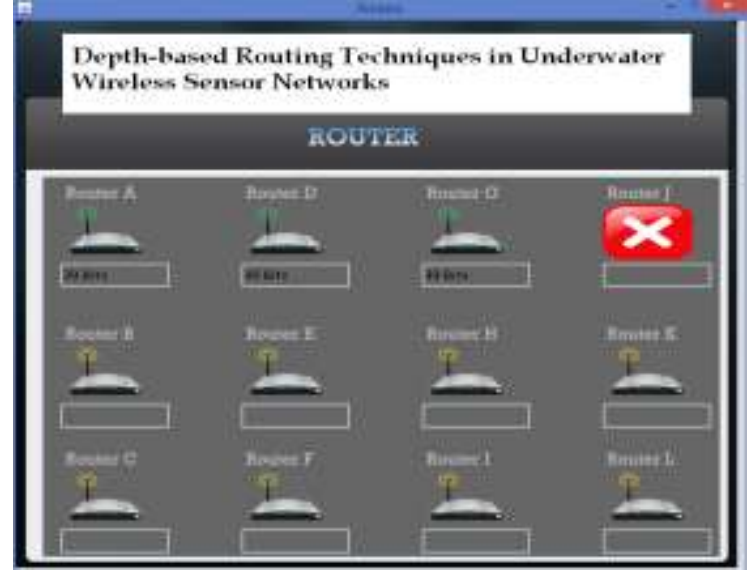

Fig. 8: Router forwarding data to sink

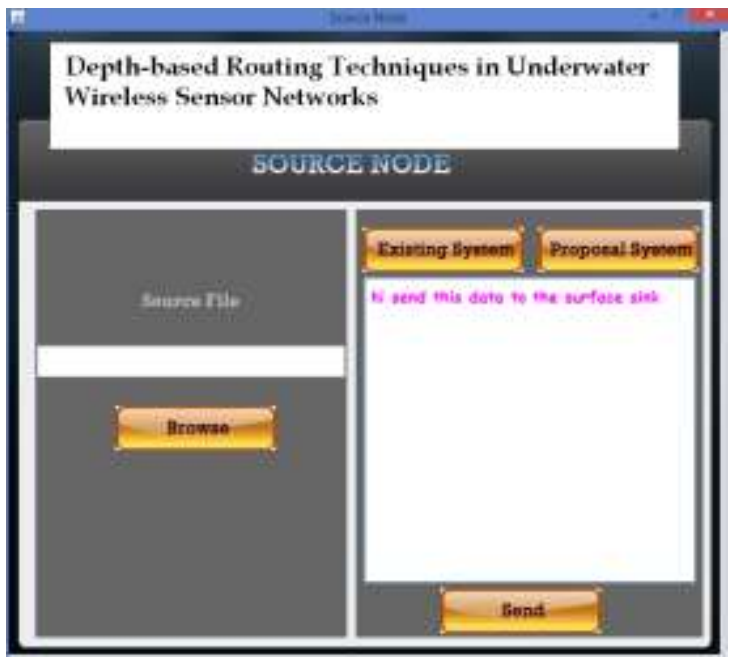

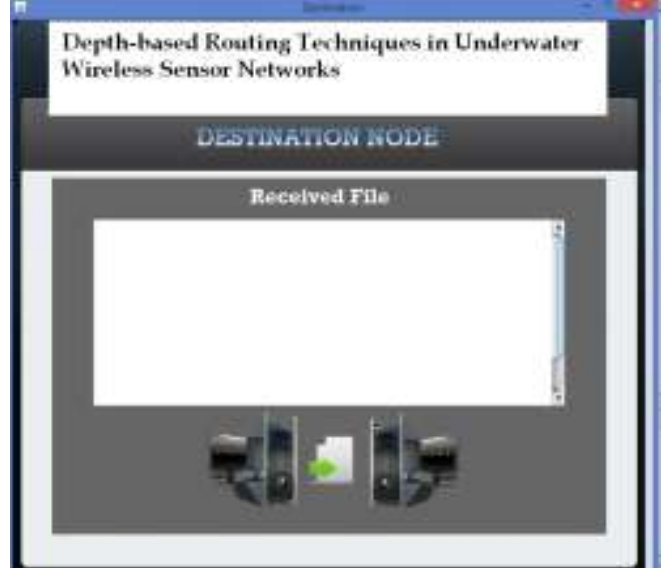

Fig. 9: No data received at the sink

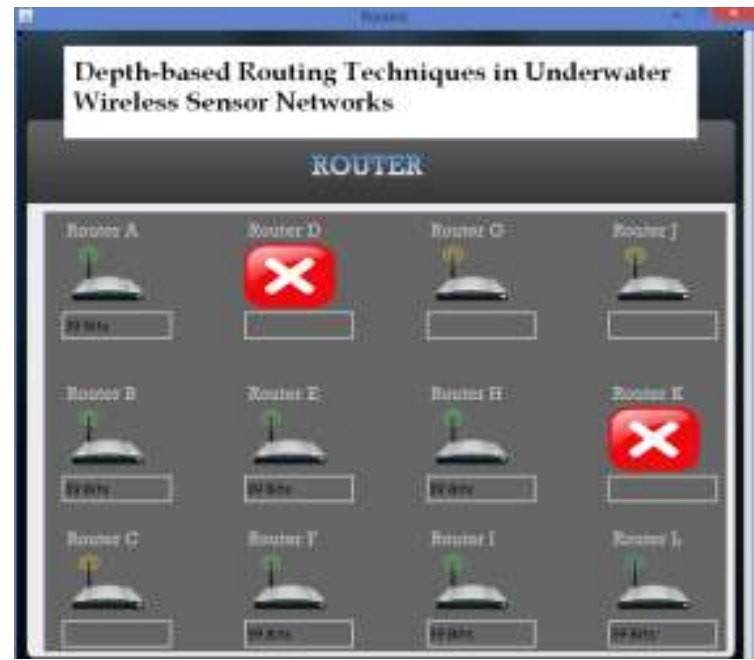

Fig. 10: Sending file to sink

Fig. 11: Router forwarding the data to sink but not in sequence

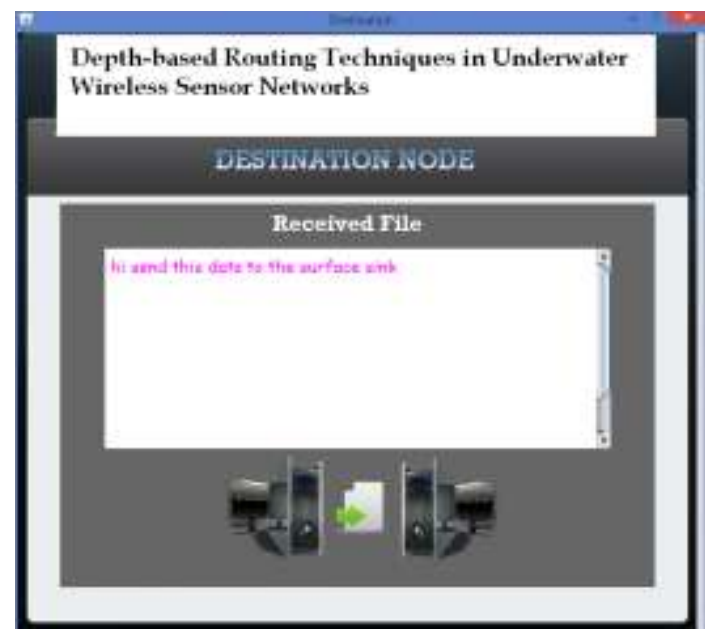

Fig. 12: Data received at the surface sink

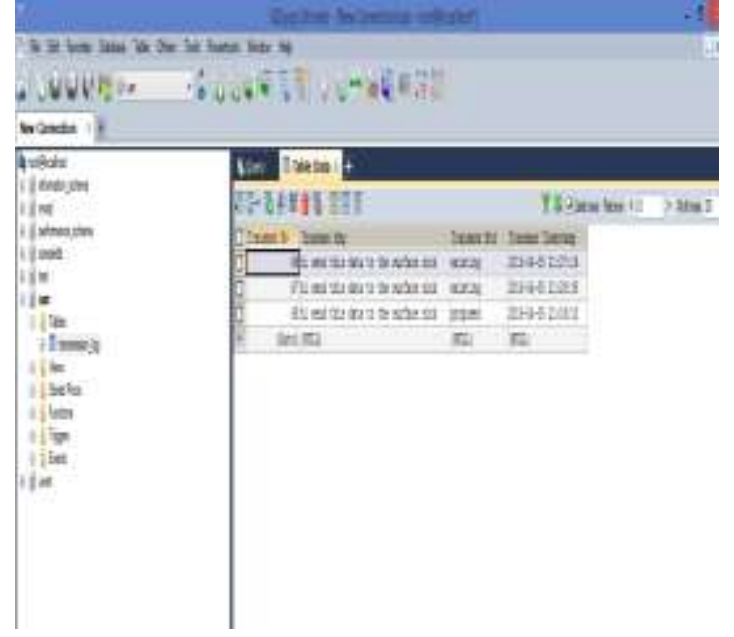

Fig. 13: Database storage

\section{Conclusion:}

In our proposed system we make use of dynamic routing and cluster head functionality. The dynamic routing concept compares the threshold value of all the nodes and then chooses the particular node at runtime irrespective of depth and forwards the data to the sink. This in turn decreases the energy consumption of node. It also minimizes the end -to - end delay especially for delay sensitive application and even in sparse condition. 
Variation in depth threshold increases the eligible neighbors thus minimizes critical data loss in delay sensitive applications. The improvement can also be made in the storage level in case of redundancy of the data.

\section{REFERENCES}

Pierre Viland, Gheorghe Zaharia and Jean Francois Helard, 2009. Coset Partitioning for the 4- PSK SpaceTime Trellis Codes, „IEEE Conference on "Signals, Circuits and Systems.

Thi Minh Hien Ngo, Gheorghe Zaharia, 2007. Stephane Bougeard and Jean Francois Helard 4-PSK Balanced STTC with two transmit antennas, IEEE Conference.

Murat Uysal and Costas N. Georghiades, 2000. Error Performance Analysis of Space-Time Codes over Rayleigh Fading Channels, Journal of Communications and Networks, 2(4): 351-356.

Magadevi, N. and Dr.V. Jawahar Senthil Kumar, 2016. Efficient Distributed Localization Algorithm for Wireless Sensor Networks, Australian Journal of Basic and Applied Sciences, 10(1): 730-734.

Pritto Paul, P., Dr.N. Sankar Ram and 3M. Usha, 2016. Symmetric Key Encryption for Secure Communication Using Wireless Hart In Wireless Sensor Networks (WSN), Australian Journal of Basic and Applied Sciences, 10(1): 625-630.

Kanagalakshmi, A., A. Mahadevan and 3A. Veeralakshmi, 2016. Design of Low Cost Microstrip Antennas for Wireless Sensor Networks, Australian Journal of Basic and Applied Sciences, 10(1): 417-424.

Senthil Murugan, M. and T. Sasilatha, 2016. A Hybrid Cryptographic algorithm design using Block and Stream cipher based Confidentiality and Integrity in Wireless Sensors Networks, Australian Journal of Basic and Applied Sciences, 10(1): 387-393.

Divya, S. and K. Stella, 2016. Priority Based Mobile Data Collection Using BiSenCar in Wireless Sensor Networks, Australian Journal of Basic and Applied Sciences Sciences, 10(1): 345-353.

Anandalatchoumy, S. and G. Sivaradje, 2016. Environment Adaptive Energy Efficient Depth Based Routing Protocol for Underwater Wireless Sensor Networks, Australian Journal of Basic and Applied Sciences, 10(12): 35-42.

Narender, M. and K.R. Anjana Devi, 2016. Wireless Sensor Network Optimization using multiple Movable Sensors for Data Gathering, Australian Journal of Basic and Applied Sciences, 10(1): 95-99.

Lissy, D. and S. Behin Sam, 2016. A Fuzzy System Based Intelligent Clustering For Wireless Sensor Networks, Australian Journal of Basic and Applied Sciences, 10(18): 251-256.

Arun Chakravarthy, R. and DR. S. Palaniswami, 2016. Efficiency With Load Balanced In Wireless Sensor Networks Improved Bat Algorithm Based Effective Stable Routing For Energy Efficiency With Load Balanced In Wireless Sensor Networks, Australian Journal of Basic and Applied Sciences, 10(8): 260-270.

Balamurugan, M. and Dr.P. Manimegalai, 2016. Implementation of Secure Fuzzy Based Authenticated Routing In Wireless Sensor Networks, Australian Journal of Basic and Applied Sciences, 10(16): 305-313.

Ayaz, Azween Abdullah, Ibrahima Faye, Yasir Batira, 2011. " An efficient Dynamic Addressing based routing protocol for Underwater Wireless Sensor Networks",computer communication-35.

Ravindranath Vadlamudi, Dr Syed Umar, 2013. "A Review of APTEEN in Wireless Sensor Networks,'IJCSET 3: 9.

Thumpi, R., R.B. Manjula, Sunilkumar S. Manvi, 2013. 'A Survey on Routing Protocols for Underwater Acoustic Sensor Networks,'-IJRTE,ISSN:22773878, 2.

Muhammad Ayaz, Imran Baig, Azween Abdullah, 2011. Ibrahima Faye, A survey on routing techniques in underwater wireless sensor networks, $1084-8045 / \$$ - see front matter \& Elsevier Ltd., doi:10.1016/j.jnca.2011.06.009.

Upena Dalal, 2009. - Wireless Communication, Oxford University Press, India.

Hai Yan, Zhijie Jerry Shi, and Jun-Hong Cui, 2010. ” DBR: Depth-Based Routing for Underwater Sensor Networks", International Conference on Industrial Mechatronics and Automation.

Jafri, M.R., S. Ahmed, N. Javaid Z. Ahmad, R.J. Quresh, 2013. ' IAMCTD: Improved Adaptive Mobility of Courier nodes in Threshold-optimized DBR Protocol for Underwater Wireless Sensor Networks'arXiv:1307.7009V1[cs 41] 61.

Javaid, N., U. Qasim, Z.A. Khan, M.A. Khan, K. Latif, A. Javaid, 2013.” On Energy Efficiency and Delay Minimization in Reactive Protocols in Wireless Multi-hop Networks",978-1-4673-6195-8/13.

Jian-feng yan, yuan-liu liu, 2011. " Improved LEACH Routing Protocol For Large Scale Wireless Sensor Networks Routing",school of computer science.

Kalpana Sharma, Neha Mittal, Priyanka Rathi, 2013.” Performance analysis of flooding and SPIN in Wireless Sensor Networks",IJFCGN- 7.

Tahir, M., N. Javaid, A. Iqbal, Z.A. Khan and N. Alrajeh, 2013. " On Adaptive Energy-Efficient Transmission in WSNs", 8.

Ian, F., Akyildiz, Dario Pompili, Tommaso Melodia, 2006. State of the Art in Protocol Research for Underwater Acoustic Sensor Networks, WUWNet06, Los Angeles, California, USA. 
Theodore, S., Rappaport, 2002. Wireless Communications: Principals and Practice, 2nd ed., Pearson Education (Singapore) Pte. Ltd.,India.

Murat Uysal, and Costas N. Georghiades, 2004. On the Error Performance Analysis of Space-Time Trellis Codes, IEEE Transactions on Wireless Communications, 3(4): 1118-1123.

Pillai, J.N. and S.H. Mneney, 2004. Adaptively Weighted Space- Time Trellis Codes, Southern African Telecommunication Networks and Application Conference.

Helmut Bolcskei and Arogyaswami J. Paulraj, 2000. Performance of space-time codes in the presence of spatial fading Correlation, IEEE Conference, 1: 687-693.

Siavash, M. Alamouti, 1998. A Simple Transmit Diversity Technique for Wireless Communications, IEEE Journal on Select Areas in Communications, 16(8): 1451-1458. 